\title{
VHF radio signal modulation classification based on convolution neural networks
}

\author{
Hao $\mathrm{Wu}^{\mathrm{a}}$, Qing Wang, Liang Zhou, and Jin Meng \\ National Key Laboratory of Science and Technology on Vessel Integrated Power System, Naval University of Engineering, \\ Wuhan, Hubei, 430033, China
}

\begin{abstract}
Deep learning architecture has been attracting increasing attention due to the successful applications in various fields. However, its application in radio system has not been well explored. In this paper, we consider the very high frequency (VHF) radio signal modulation classification based on convolution neural networks $(\mathrm{CNN})$. The main principle of $\mathrm{CNN}$ is analysed and a five-layer $\mathrm{CNN}$ model is built. The proposed CNN-based modulation classification method is proved useful for simulated radio signals generated by MATLAB, that the overall classification accuracy is high even in low SNR. In addition, the proposed $\mathrm{CNN}$-based method is used for real VHF radio signals, and the key factors effecting the classification accuracy are analysed.
\end{abstract}

\section{Introduction}

Automatic modulation classification (AMC) has been a hot research topic of communication system in many years. AMC plays an important role in military and cognitive radio applications, especially in very high frequency (VHF) bands. For example, in military affairs, AMC is the precondition of disturbing or intercepting the enemy communication. Once the modulation type of signal is known, the modulation parameters can be estimated, and the investigation and anti-reconnaissance strategies can be formulated. In cognitive radio, AMC allows for dynamically changing modulation without increasing communication overhead.

Previously, AMC has been mainly accomplished by the feature-based methods [1] or the likelihood-based methods [2]. Feature-based methods require the expert features of signal, such as instantaneous amplitude, phase, constellation diagram, cyclic spectrum and cumulants. Likelihood-based methods, including average likelihood ratio test (ALRT), general likelihood ratio test (GLRT) and hybrid likelihood ratio test (HLRT) methods, require the prior information or specific parameters of signal. It should be noted that, traditional feature-based or likelihood-based methods can achieve high classification accuracy under certain conditions. However, they require lots of knowledge and expert design of signals, which lead to high computational complexity in practice. Moreover, with the development of radio technologies and communication systems, modulation mode of signals become complex and traditional methods are limited in some non-ideal environment with low signal-to-noise ratio.

Recently, deep learning (DL) architecture has been attracting increasing attention due to the successful applications in various fields, such as natural language processing (NLP) [3], computer vision (CV) [4], bioinformatics [5] and economics [6]. DL is an emerging field of machine learning (ML) thanks to the improvement in hardware and big data analysis [7]. Compared to classical ML, DL can use more numerous successive layers of non-linear processing units to extract high-order characteristics of data. In general, DL is good at extracting high-order features and is the procedure for mapping input data to output data of deep neural networks.

Many researchers are trying to extend DL to new field, obviously including AMC [8-13]. In [8], the authors propose a DL architecture based on four-layer convolutional neural networks for modulation recognition. It can effectively identify 11 analog and digital modulation signals. In [9], three convolutional neural networks are established based on temporal IQ data, amplitude/phase data and frequency domain data, respectively, and the proposed method can reach modulation classification accuracy of $95 \%$ even in low SNR. Based on [8], the authors in [10] propose a new DL architecture using residual neural network (RNN) for 24 modulation signals classification, and the trained deep networks can be effectively transferred to over-the-air datasets. In [11], a modulation recognition algorithm based on antinoise processing and deep sparse-filtering convolutional neural network (AN-SF-CNN), is proposed for modulation classification of VHF radio signals. In [12], a spectrum monitoring framework based on deep CNN model is presented, and the classification accuracy can reach $99.6 \%$. In [13], signal constellation diagram is introduced and two new CNN-based DL models, AlexNet and GoogLeNet, are used for modulation classification.

In this paper, we consider the VHF radio signal modulation classification problem. Based on the DL

\footnotetext{
a Corresponding author: wowhow@163.com
} 
architectures, a multi-layer $\mathrm{CNN}$ model is built for addressing this issue. The proposed CNN model consists of five layers and is proved useful for simulated VHF radio signals generated by MATLAB, that the overall classification accuracy is high even in low SNR. In addition, we focus on the modulation classification of real VHF radio signals. Experiments show that the classification accuracy of real radio signal is not very satisfied, and the key factors effecting the accuracy are given.

The rest of this paper is structured as follows. In section II, the main principle of CNN is analysed and the structure of the CNN model for VHF radio signal classification is given. Section III provides simulation and experiment to verify the proposed CNN model, and Section IV gives the conclusions.

\section{The structure of CNN}

Constructing a deep neural network with multi-layers and multi-units, each layer has the function of extracting a large number of data, which is the core idea of DL. Technically, deep neural network aims at extracting features from raw input data, which is so-called datadriven feature extraction. In traditional artificial feature extraction method, detection and extraction features are complex and may be inaccurate. In contrast, deep neural network can extract complex high-order features by assembling simple features.

DL architecture has many models, such as CNN, restricted Boltzmann machines (RBM), and RNN. In this paper, considering the characteristics of VHF signals, CNN model is applied for modulation classification.

As shown in Fig. 1, CNN contains three types of layers: convolutional layer, pooling layer and fully connected layer. The convolutional layer is the core of convolutional neural network that consumes the most computation, the pooling layer replaces the output of a location with the nearby relevant statistics output, and the fully connected layer has full connections to all activations. Specifically, the input feature image $x_{i}^{(i-1)}$ is convoluted with the convolutional kernel $K_{i j}$, and then the output feature image $y_{j}^{(l)}$ of the $l$ th convolutional layer is obtained as follows

$$
y_{j}^{(l)}=g\left(\sum_{i \in M_{j}} K_{i j} \otimes x_{i}^{(l-1)}+b_{j}\right)
$$

(1)

where $\otimes$ denotes the convolution operation, $b_{j}$ denotes the bias, $M_{j}$ denotes the set of feature map, and $g(\cdot)$ denotes the nonlinear function. Generally, the nonlinear function $g(\cdot)$ can be $g(x)=\tanh (x), \quad g(x)=\left(1+e^{-x}\right)^{-1}$ or $g(x)=\max (0, x)$.

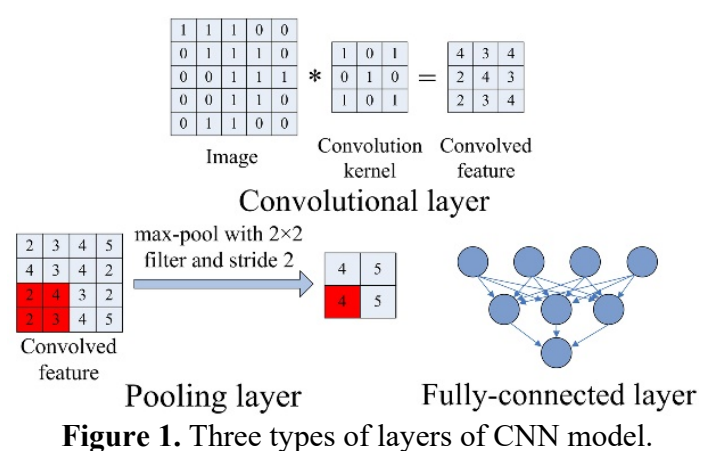

Therefore, the learning process of $\mathrm{CNN}$ can be divided into two steps, feed-forward step and back-propagation step. In feed-forward step, the sum of the products of the weights and the inputs is calculated in each node. If the value is above some threshold, the neuron fires and takes the activated value, otherwise it takes the deactivated value. In back-propagation step, gradient descent optimization algorithm is commonly used for adjusting the weight of neurons by calculating the gradient of the loss function.

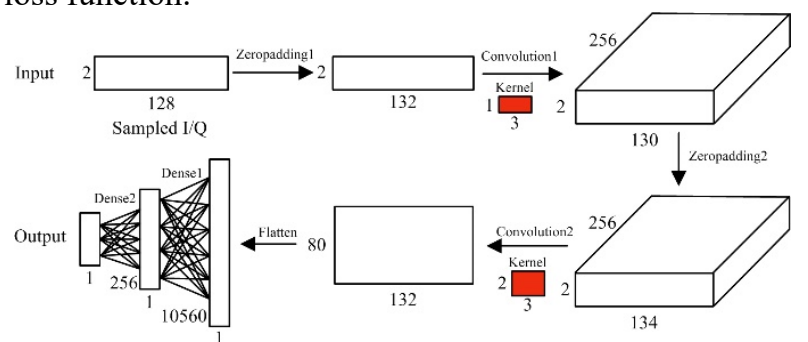

Figure 2. The main structure of CNN for VHF signal modulation classification.

Fig. 2 shows the main structure of the proposed CNN for VHF signal modulation classification. As shown in Fig. 2, we treat the complex raw I/Q data of VHF baseband signal as the input $2 \times N$ vectors, the length is set as $N=128$, and thus the input size of CNN is $2 \times 128$. The proposed CNN contains two convolutional layers and two fully connected layers. The size of the two convolution kernel is $1 \times 3$ and $2 \times 3$, respectively, and the size of the two fully connected layers is $10560 \times 1$ and $256 \times 1$, respectively.

The relationship of each layer is given as follow. First, to make the convolution operation easier, a larger feature image of size $2 \times 132$ is obtained by zeropadding. Second, 256 convolution kernels of size $1 \times 3$ are used, and the size of the convolutional layer is $256 \times 2 \times 130$. Next, a larger feature image of size $256 \times 2 \times 134$ is obtained by zeropadding, 80 convolutional kernels of size $2 \times 3$ are used, and the size of the convolutional layer is $80 \times 132$. Then, the convolutional layer is converted to flatten layer of size $10560 \times 1$, and lastly the output is obtained by two fully connected layers. Additionally, the activation function of the convolutional layer is rectified linear unit (ReLU) and the activation function of the last fully connected layer is Softmax, respectively. The main parameters of the proposed CNN are summarized in Table 1. 
Table 1. The main parameters of $\mathrm{CNN}$

\begin{tabular}{lllll}
\hline Layer & Input size & Output size & Parameters & A-fct. \\
\hline Conv. & $2 \times 132$ & $256 \times 2 \times 130$ & $\begin{array}{l}256 \text { kernels } \\
\text { of size } 1 \times 3\end{array}$ & ReLU \\
Conv. & $256 \times 2 \times 134$ & $80 \times 132$ & $\begin{array}{l}80 \text { kernels } \\
\text { of size } 2 \times 3\end{array}$ & ReLU \\
FC & $10560 \times 1$ & $256 \times 1$ & 256 neurons & ReLU \\
FC & $256 \times 1$ & $10 \times 1$ & 10 neurons & Softmax \\
\hline
\end{tabular}

\section{Simulation and experiment}

In this section, we provide simulation and experiment to illustrate the performance of the proposed CNN model for VHF signal classification. We use computation library Theano [14] as the DL framework, due to its modularity with Python support. Based on Theano, an open source library written in Python, Keras, is utilized.

Firstly, we apply CNN model for the following simulated signals across 10 modulations (BPSK, QPSK, 8PSK, 4ASK, 8ASK, 2FSK, 4FSK, 8FSK, 16QAM, 64QAM) extensively used in VHF band. One-hot encoding is used to create 10 class labels corresponding to 10 modulations, and the task of modulation classification is then a 10-class classification problem. The data vectors are all generated by MATLAB 2017a, well labelled in 128 sample batches, and the SNR is set to $40 \mathrm{~dB}$.

We train our CNN model with $2000 \times 10$ sample training set in batch sizes of 128 , and test it with $500 \times 10$ sample testing set. The train loss and validation loss are shown in Fig. 3.

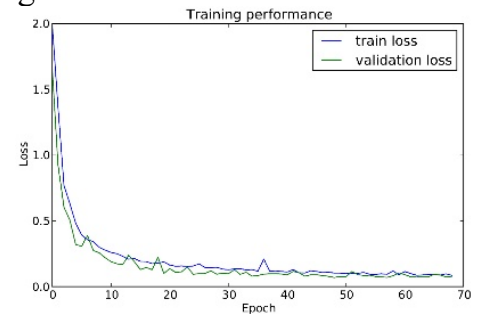

Figure 3. Train loss and validation loss of $\mathrm{CNN}$ model.

It is obvious that, the training process is slightly unstable and is finished at about 68 epochs under the preset thresholds. Fig. 4 shows the modulation classification accuracy of 10 modulation signals. The overall classification accuracy can reach $99 \%$. However, $4 \mathrm{ASK} / 8 \mathrm{ASK}$ and $4 \mathrm{FSK} / 8 \mathrm{FSK}$ are partly confused, due to the similarity of signals in baseband.

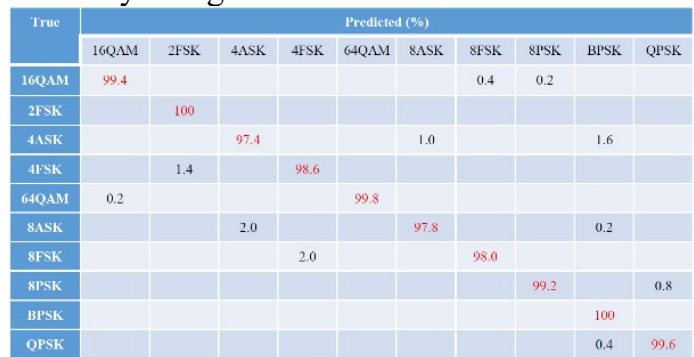

Figure 4. Classification accuracy of simulated signals.

It should be noted that, the train data and test data used in Fig. 4 is obtained at high SNR of $40 \mathrm{~dB}$. Thus we present the effects of low SNR on classification accuracy.

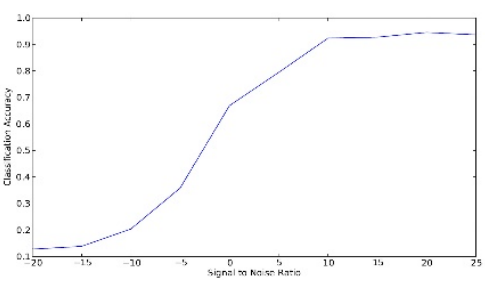

Figure 5. Classification accuracy versus signal to noise ratio.

Fig. 5 plots classification accuracy versus SNR. As SNR increases from $-20 \mathrm{~dB}$ to $20 \mathrm{~dB}$, the classification accuracy first quickly increases, and then slightly increases, which is consistent with our theoretical analysis. When $\mathrm{SNR}=10 \mathrm{~dB}$, the overall classification accuracy can reach $95 \%$, therefore, the $\mathrm{CNN}$-based modulation classification method is efficient even in low SNR.

Next, we apply CNN model for a real VHF radio and the corresponding hardware platform is built. The VHF radio in this experiment has two modes, including data mode and speech mode, at different frequency. In data mode the following signals across 5 modulations (BPSK, QPSK, 8PSK, MSK and TCM) are used, and in speech mode the following signals across 5 modulations (AM, FM, DS, FH and DF) are used.

Fig. 6 shows the flowchart of modulation classification of real VHF radio, and Fig. 7 shows the corresponding hardware platform. First, modulation signals of VHF radio are generated by communication manager of PC or microphone, and we sample it using an oscilloscope. Next, the baseband I/Q data is obtained by digital down conversion, and then the raw I/Q data is transmitted to a PC, which is embedded with DL framework. At last, modulation classification can be finished by the proposed CNN model.

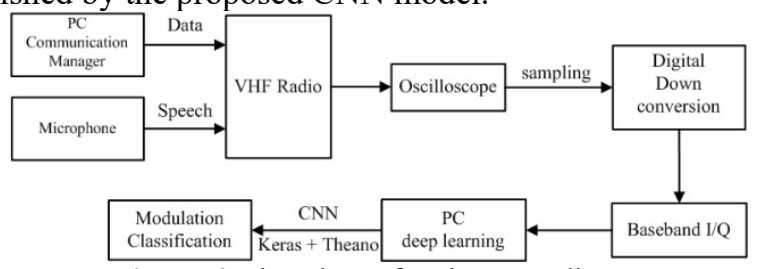

Figure 6. Flowchart of real VHF radio.

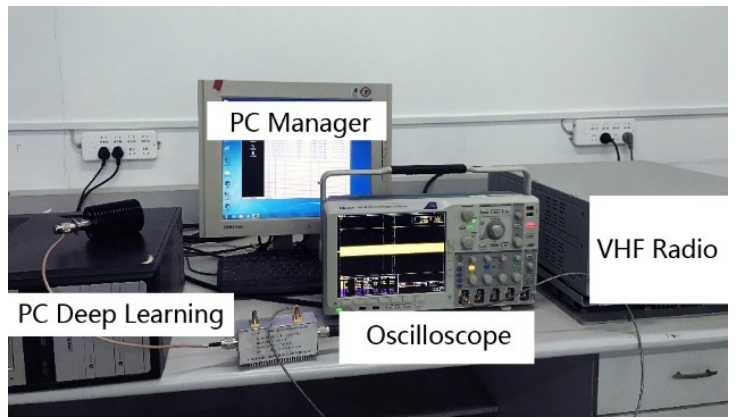

Figure 7. Hardware platform of real VHF radio.

In data mode, we train our CNN model with $6000 \times 5$ sample training set in batch sizes of 128 , and test it with $600 \times 5$ sample testing set. The train/validation loss is shown in Fig. 8(a), and the classification confusion matrix is shown in Fig. 8(b), respectively. Note that the darker the block in confusion matrix, the higher the corresponding classification accuracy. It is obvious that TCM and 8PSK signals can be classified, while BPSK, QPSK and MSK signals are seriously confused, the 
overall classification accuracy is only $55 \%$. The reason may be that train data set is too small and the convolutional neural network is too simple, thus overfitting of training model is occurred as shown in Fig. 8(a).

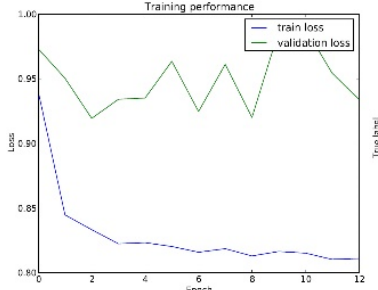

(a)

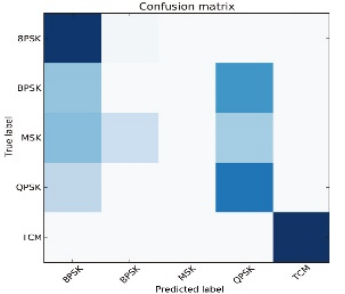

(b)
Figure 8. Performance of real VHF radio in data mode.

(a) Train loss and validation loss. (b) Confusion matrix.

In speech mode, we train our CNN model with $6000 \times 5$ sample training set in batch sizes of 128 , and test it with $600 \times 5$ sample testing set. The train/validation loss is shown in Fig. 9(a), and the classification confusion matrix is shown in Fig. 9(b), respectively. It is obvious that AM and FH signals can be classified, while DF, DS and FM signals are seriously confused, the overall classification accuracy is $76 \%$.

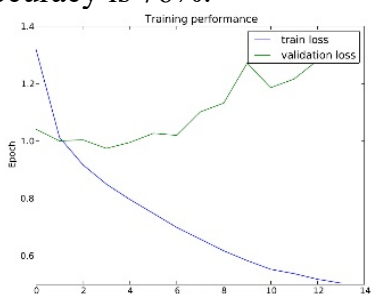

(a)

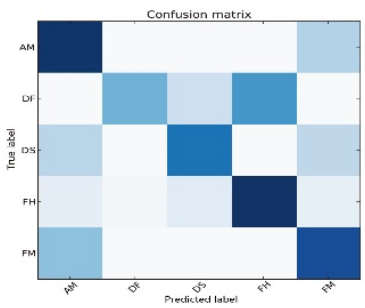

(b)
Figure 9. Performance of real VHF radio in speech mode.

(a) Train loss and validation loss. (b) Confusion matrix.

Interestingly, if we increase the training data to $10000 \times 5$ sample training set in speech mode, the overall classification accuracy can rise to $84 \%$, which means that the size of training data has a significant effect on the modulation classification.

As shown above, the classification accuracy of real VHF radio signals is not very satisfied, due to frequency offset, symbol rate, modulation coefficient, roll-off factor and other noise of real radio are unknown, the synchronization parameters estimation is very difficult, and the proper data for training set is difficult to collect. Therefore, synchronization parameter estimation is a key point for modulation classification of real radio.

\section{Conclusions}

In this paper, we propose a CNN-based method for VHF radio signal modulation classification. We analyse the principle of $\mathrm{CNN}$, and build a five-layer model with two convolutional layers and two fully connected layers. In simulation, we consider the classification of 10 simulated VHF signals generated by MATLAB, and the overall classification accuracy can reach $95 \%$ even in low SNR. Finally, we focus on the classification of modulation signals of real VHF radio. The overall classification accuracy is $55 \%$ in data mode and $76 \%$ in speech mode, respectively, due to the effect of frequency offset, phase noise and other noise. Possible future research might concern the problem of synchronization parameter estimation of real radio systems, and the effective way for extracting useful training data.

\section{Acknowledgement}

This work was supported by the National Natural Science Foundation of China under Grants 61801502 and 71801220 .

\section{References}

1. W.C. Headley, J.D. Reed and C.R.C. Silva, "Distributed cyclic spectrum feature-based modulation classification", 2008 IEEE Wireless Communicaitions and Networking Conference, Las Vegas, USA, March 2008, pp. 1200-1204.

2. R. Ali, K. Ii-Min, K. Dong, C. Francois and I. Robert, "Likelihood-based modulation classification for multiple-antenna receiver", IEEE Transactions on Communications, vol. 61, no. 9, pp. 3816-3829, 2013.

3. N. Jean, M. Burke, M. Xie, W. M. Davis, B. Lobell, and S. Ermon, "Recent trends in deep learning based natural language processing”, IEEE Computational Intelligence Magezine, vol. 13, no. 3, pp. 55-75, 2018.

4. A. Voulodimos, N. Doulamis, A. Doulamis, E. Protopapadakis. "Deep learning for computer vision: a brief review", Computational Intelligence and Neuroscience, pp. 1-13, 2018.

5. K. Lan, D, Wang, S. Fong, L. Liu, K. Wong and N. Dey, "A survey of data mining and deep learning in bioinformatics", Journal of Medical Systems, vol. 42, no. 8, pp. 1-20, 2018.

6. N. Jean, M. Burke, M. Xie, W. M. Davis, B. Lobell, and S. Ermon, "Combing satellite imagery and machine learning to predict poverty", Science, vol. 353, no. 6301, pp. 790-794, 2016.

7. Z. Zheng, T. Huang, H. Zhang, S. Sun, J. Wen, and P. Wang, "Towards a resource migration method in cloud computing based on node failure rule", Journal of Intelligence and Fuzzy Systems, vol. 31, no. 5, pp. 2611-2618, 2016.

8. T. J. OShea, J. Corgan, and T. C. Clancy, "Convolutional radio modulation recognition networks", 2016 International Conference on Engineering Application of Neural Networks, Springer International Publishing, pp. 213-226, 2016.

9. M. Kulin, T. Kazaz, I. Moerman, and E. De Poorter, "End-to-end learning from spectrum data: a deep learning approach for wireless signal identification in spectrum monitoring applications", IEEE Access, vol. 6, pp. 18484-18501, 2018.

10. T. J. OShea, T. Roy, and T. C. Clancy, "Over the air deep learning based radio signal classification”, IEEE Journal of Selected Topics in Signal Processing, vol. 12, no. 1, pp. 168-179, 2018.

11. R. Li, L. Li, S. Yang and S. Li, "Robust automated VHF modulation recognition based on deep 
convolutional neural networks", IEEE Communications Letters, vol. 22, no. 5, pp. 946-949, 2018.

12. S. Ahmed, P. Francisco, A. Jerome, Z. Yi and D. Linda, "Spectrum monitoring for radar bands using deep convolutional neural networks", 2017 IEEE Global Communications conference, Singapore, Dec. 2017, pp. 1-6.

13. S. Peng, H. Jiang, H. Wang, H. Alwageed, Y. Zhou, M. M. Sebdani and Y.D. Yao, "Modulation classification based on signal constellation diagrams and deep learning", IEEE Transactions on Neural Networks and Learning Systems, pp. 1-10, 2018, doi: 10.1109/tnnls.2018.2850703.

14. J. Bergstra, F. Bastien, O. Breuleux and P. Lamblin, "Theano: deep learning on GPUs with python", Journal of Machine Learning Research 1, pp. 1-4, 2011. 